\title{
Commentary
}

\section{Targeting Stress Pathophysiology to Improve Alcoholism Relapse Outcomes}

\author{
Verica Milivojevic ${ }^{1,2}$ and Rajita Sinha*,1,2,3 \\ 'Yale Stress Center, Yale University School of Medicine, New Haven, CT, USA; ${ }^{2}$ The Department of Psychiatry, Yale University School of Medicine, \\ New Haven, CT, USA; ${ }^{3}$ The Department of Neuroscience, Yale University School of Medicine, New Haven, CT, USA
}

Neuropsychopharmacology (2017) 42, 987-988; doi:I0.I038/npp.20 I6.234; published online 26 October 2016

Excessive alcohol use is one of the top behavioral causes of global disease burden. A major factor contributing to this burden is the alcohol relapse risk during treatment and recovery from alcoholism. Clinical studies suggest that stress is one of the key factors that contributes to these high-relapse rates (Blaine et al, 2016). Changes in hypothalamic pituitary adrenal (HPA) axis responses, altered and blunted amygdala response to fear/threat potentiated startle in heavy drinkers compared with light social drinkers and autonomic imbalance in sympathetic/parasympathetic systems, have been reported with increased alcohol use (Sinha et al, 2011a). Furthermore, acute alcohol intake stimulates the HPA axis and increases cortisol levels (see Blaine et al (2016) for review), but chronic alcohol abuse is associated with blunted stress cortisol responses (Lovallo et al, 2000). In addition, alcohol withdrawal is associated with elevated basal cortisol (Blaine et al, 2016).

In our laboratory, we have used personalized guided stressful and drug/alcohol cue-related imagery to provoke this stress and high-craving dysfunction in early abstinent alcohol-dependent (AD) men and women. We compared a group of 4-week abstinent and recovering treatment engaged $\mathrm{AD}$ individuals to healthy controls across three imagery conditions (stress, alcohol cue, and neutral/relaxing) and assessed subjective and neuroendocrine responses at baseline, immediately following imagery exposure and at additional post-imagery time periods. We found significant HPA axis dysregulation, which was marked by higher basal ACTH levels and blunted stress- and cue-induced ACTH and cortisol responses in the $\mathrm{AD}$ patients compared with the control group (Sinha et al, 2011b). The AD individuals also displayed higher anxiety, and greater stress- and cue-induced alcohol craving compared with the control group. Importantly, we found that individuals with high provoked alcohol craving but also high cortisol and greater cortisol/ACTH ratios (which is a measure of sensitivity of the adrenal glands

\footnotetext{
* Correspondence: Professor R Sinha, Yale Stress Center, Yale University School of Medicine, Suite 209, 2 Church Street South, New Haven, CT 065 19, USA, Tel: + I 203737 5805, Fax: + I 203737 1272, E-mail: Rajita.sinha@yale.edu

Accepted article preview online 7 October 2016
}

to release cortisol in response to the ACTH signal) relapsed more quickly after discharge from inpatient treatment. In fact, high cortisol/ACTH ratios more than doubled the risk of shorter time to future relapse (Sinha et al, 2011b). These findings suggest that both craving and the stress-related HPA axis dysfunction may play a role in high-relapse rates in alcoholism and that targeting this stress pathophysiology pharmacologically could improve alcohol relapse rates.

Vasopressin (arginine vasopressin; AVP) is one of the two major neuropeptides that regulates the HPA axis. Together with CRF, AVP stimulates the release of ACTH from the pituitary via the AVP $1 \mathrm{~b}$ (V1b) receptor. Preclinical studies have found that pharmacological blockade of the V1b receptor results in anxiolytic and antidepressant effects and decreases in alcohol intake in alcohol-dependent rats (Edwards et al, 2012). Such findings suggest that a V1B receptor antagonist may block pituitary activation of the HPA axis and may serve as a potential treatment target for alcohol use disorders.

In this issue, Ryan et al (2016) tested this possibility and report interesting findings from a multi-site study evaluating a novel V1b antagonist compound, ABT-436, in the treatment of alcoholism. AD patients were titrated to $800 \mathrm{mg} /$ day of ABT-436 or placebo in a 12-week doubleblind trial with $150 \mathrm{AD}$ men and women. Subjects were carefully screened to rule out medical and psychiatric conditions requiring medications, except for stable use of antidepressants. Importantly, the authors also conducted an ACTH stimulation test at screening, week 5 and end of the study, to stratify patients on high anxiety and high cortisol responses and also to assess medication effects on possible HPA axis suppression. A priori primary outcomes were percentage of heavy drinking days and secondary outcomes of other drinking measures as well as alcohol craving, number of alcohol-related consequences, and number of cigarettes smoked per day were assessed.

The authors reported ABT-436 to be generally safe and well tolerated, and the percentage of heavy drinking days to be lower in the ABT-436 group compared with placebo, but this effect on primary outcome was not significant. Interestingly, percentage of heavy drinking days was 
significantly lower than placebo only in the first week of the maintenance period. On the other hand, ABT-436 showed greater efficacy on the percentage of drinking days in participants with greater levels of baseline stress, as assessed by the POMS Tension-Anxiety, peak cortisol levels during the ACTH stimulation test, and Spielberger Trait Anxiety Index scores. In terms of secondary outcomes, the ABT-436 group had significantly greater percentage of days of alcohol abstinence and significantly reduced number of cigarettes smoked per week compared with placebo averaged across the maintenance period.

This study is the first clinical trial of ABT-436 in AD patients and highlights some important aspects of pharmacologically targeting the stress system dysfunction in alcoholism. The authors are to be commended on carefully characterizing patients not only on baseline levels of stress and anxiety but also on peak cortisol levels in the ACTH stimulation test and showing that such stratification was important in treatment response. However, previous human studies assessing the biological stress axis show a specific disruption of the HPA axis with higher tonic cortisol (basal) and blunted cortisol responses (phasic) in recently drinking and early abstinent/withdrawn AD patients (Sinha et al, 2011b). Thus, while targeting patient subgroups with stress system dysfunction is critical to improve alcoholism outcomes, it may be important to target the HPA axis pathophysiology seen in $\mathrm{AD}$ patients more specifically. That is, as blunted cortisol response to stress and challenge is seen in $\mathrm{AD}$, compounds that suppress the HPA axis will like suppress both basal tonic and phasic stress-induced cortisol response, the latter already being suppressed in $\mathrm{AD}$ patients, a response that has associated with higher relapse and alcohol intake. This would suggest that those patients vulnerable to blunted stress responses and those continuing to use alcohol even at lower levels (that is non-abstinent treatment goals) may not respond to ABT-436. Indeed, the authors' finding of positive medication effects for percent heavy drinking days in the first maintenance week suggests that blockade of AVP signaling may have been most effective in suppressing the high basal HPA axis levels during early abstinence/acute withdrawal. Their findings support further study to carefully assess ABT-436 versus placebo during acute withdrawal and in early recovery to further evaluate its effects on the tonic and phasic HPA axis responses in early abstinent AD individuals.
The AVP system presents a potentially promising target for medication development in alcohol dependence, especially in subpopulations of individuals who display biological phenotypes of tonic hyper-reactivity to stress, such as during the acute withdrawal phase, and for those who due to health and other reasons have a strong commitment to abstinence. With greater research to understand its effects in patients and further testing, it certainly seems possible that vasopressin antagonists may have a role in the armamentarium of effective medications for treating alcoholism among specific subgroups of $\mathrm{AD}$ individuals.

\section{FUNDING AND DISCLOSURE}

Dr. Sinha is on the Scientific Advisory Board for Embera Neurotherapeutics and has received compensation for consultation. This research was supported by grants from the NIH National Institute of Alcohol Abuse and Alcoholism (NIAAA), grant R01-AA020504. Dr. Milivojevic declares no potential conflict of interest.

\section{REFERENCES}

Blaine SK, Milivojevic V, Fox H, Sinha R (2016). Alcohol effects on stress pathways: impact on craving and relapse risk. Can J Psychiatry 61: 145-153.

Edwards S, Guerrero M, Ghoneim OM, Roberts E, Koob GF (2012). Evidence that vasopressin $\mathrm{V} 1 \mathrm{~b}$ receptors mediate the transition to excessive drinking in ethanol-dependent rats. Addict Biol 17: $76-85$.

Lovallo WR, Dickensheets SL, Myers DA, Thomas TL, Nixon SJ (2000). Blunted stress cortisol response in abstinent alcoholic and polysubstance-abusing men. Alcohol Clin Exp Res 24: 651-658.

Ryan ML, Falk DE, Fertig JB, Rendenbach-Mueller B, Katz DA, Tracy KA et al (2016). A phase 2, double-blind, placebocontrolled randomized trial assessing the efficacy of ABT-436, a novel V1b receptor antagonist, for alcohol dependence. Neuropsychopharmacology (this issue).

Sinha R, Shaham Y, Heilig M (2011a). Translational and reverse translational research on the role of stress in drug craving and relapse. Psychopharmacology (Berl) 218: 69-82.

Sinha R, Fox HC, Hong KI, Hansen J, Tuit K, Kreek MJ (2011b). Effects of adrenal sensitivity, stress- and cue-induced craving, and anxiety on subsequent alcohol relapse and treatment outcomes. Arch Gen Psychiatry 68: 942-952. 